\title{
A DATABASE SYSTEM FOR GEOCHEMICAL, ISOTOPE HYDROLOGICAL, AND GEOCHRONOLOGICAL LABORATORIES
}

\author{
Axel Suckow \\ Institute for Joint Geoscientific Research (GGA), S3: Geochronology and Isotope Hydrology, Stilleweg 2, Hannover, \\ Germany. Email: Axel.Suckow@gga-hannover.de. \\ Ingolf Dumke \\ Federal Institute for Geosciences and Natural Resources (BGR), Stilleweg 2, Hannover, Germany
}

\begin{abstract}
We present a data model designed for laboratories in which many different methods are used. The main feature of the model is the recursive relationships of data records in the subsample table. This makes it possible to model all steps from the preparation of the sample to the final value and enables the raw data to be stored together with the final values, even if many preparation steps or many subsamples are involved. We show ways to adopt this basic model to laboratories with many years of data. The data model is extended to include laboratory records and attribute data, e.g. the geographical coordinates of the sampling site or links to the various projects for which the samples are used.
\end{abstract}

\section{INTRODUCTION}

Online measurement and digital storage of data have become a prerequisite for all laboratories. Nearly all commercial devices for chemical, isotope, and radiometric analyses include more or less well-designed database software. Storage of the primary data for many years is one of the basics of good scientific practice (DFG 1998, DCSD 1995).

Nevertheless, a modern laboratory needs a common database for all of the methods used in that laboratory. Some of the benefits of such a system are better organization and linkage of data obtained using different methods, documentation of all laboratory steps as required for ISO 9001, less workload and fewer errors, for example, due to retyping of data. Additionally, it permits standardized postprocessing and correction of the results, allows the sample data to be related to global standards, as well as provides up-to-date quality assurance data. For laboratories with data from a long period of time, this database, linking the different methods and relating them to attribute data, e.g., the geographical origin of the samples, is often the only way to enable rapid access to earlier data. If the database system is well adapted to the problems the laboratory deals with, the first modeling of the data can be done using the database software: examples are calculation of sedimentation or bioturbation rates using radionuclide-excess data or groundwater model ages (e.g. $\mathrm{CFCs}, \mathrm{T} /{ }^{3} \mathrm{He}$, and ${ }^{14} \mathrm{C}$ ).

Relational database architecture is standard for all database software. The most important and critical step in setting up a laboratory database is the design of the relational data model. It can be stated that every hour invested in the design of the data model saves hundreds of hours for writing the code for the user interface and thousands of hours during use of the database system. A flexible data model that can be used for the laboratory procedures in geochronology, isotope hydrology and geochemistry is presented here. In contrast to other laboratory database systems (Coplen 1998), any number of parameters and instruments can be used. Additionally, any number of conversions of samples into different chemical states or into any number of subsamples can be described. It uses state-of-the-art methods to enforce data integrity at the lowest possible hierarchical level, using primary and foreign keys, constraints and rules at the hierarchical level of tables, or uses triggers and SQL procedures to enforce procedural integrity. The data definition and code for the procedures uses the SQL language and, therefore, can be ported to any high-level database server software, like Oracle, RDB or MS SQL server. A user-friendly graphical user interface (GUI) enabling fast data input and retrieval was designed as well, but is not the subject of this paper.

(C) 2001 by the Arizona Board of Regents on behalf of the University of Arizona Radiocarbon, Vol 43, Nr 2A, 2001, p 325-337

Proceedings of the 17 th International ${ }^{14} \mathrm{C}$ Conference, edited by I Carmi and $\mathrm{E}$ Boaretto 


\section{Design of the Data Model Core}

Figure 1 shows the four tables of the core of the database model. The first table (tblSamplings) contains the data about the sampling action in the field. The second table contains the data on the original samples and any subsamples (tblSubSamples in Figure 1). Hereafter, the term "subsample" includes the original sample; this is because the original sample is included in the same table as the subsamples and assigned a precursor marker equal to "Null". The method used for preparing a subsample is given in the third table (tblProcedures in Figure 1). Subsamples can be produced during the sampling action in the field (e.g. bottles of water for the tritium, stable isotope and radiocarbon analyses and the filtered and acidified samples for geochemical analysis) or during laboratory processing (e.g. taking aliquots, sieving, preparation of a counting gas).

The measured values are stored in the fourth table (tblValues in Figure 1). The methods used for the measurements are stored in tblProcedures. The values table is mainly for storing only the numerical values and will have the largest number of rows of all the tables in the data model. It is therefore necessary to keep the number of columns ("fields") in this table small and to avoid strings and redundant information wherever possible. Therefore, the names of the parameters (e.g. "tritium", "180", " $\left.{ }^{14} \mathrm{C} "\right)$ and units (e.g., ppm, TU, pMC, \%o) are stored in the procedures table. Also detailed information about the procedure itself is stored in the table tblProcedures: the field imManual can contain a whole text file (e.g. MS Word) with a certain laboratory procedure according to ISO 9001.

Note that a 1:n relation exists between the procedures in tblProcedures and the subsamples in tblSubSamples (many subsamples can be produced by the same method), between procedures and values (many measurement values can be produced using the same procedure) and between subsamples and values (methods like gamma spectrometry and ICP-MS result in many values for the same subsample, and measurements can be repeated).

It is possible for recursive relationships to exist in the subsample table: many subsamples can be produced from one (sub)sample (the "precursor") using standard laboratory methods. The most straightforward example is the production of aliquots. Another example in geochemical laboratories is the sieving of sediment to obtain grain-size fractions for later chemical analysis. An example in an isotope hydrology laboratory is electrolytic tritium enrichment, resulting in a subsample with a higher tritium content than the precursor. In a radiocarbon laboratory with proportional counters, dilution with a "dead" counting gas of a sample that is too small for the counter is another example.

This data model has the following advantages:

1. Scalability. Since the number of rows in the table for procedures is limited only by the available storage space, the system can handle a virtually infinite number of methods to process samples, to produce subsamples and data.

2. Effective use of storage space. Empty cells in the table of values are avoided. If a laboratory procedure generates only one value for a subsample or 100 , only the amount of storage space necessary is used.

3. Flexibility. The data model can be adapted to many kinds of laboratories and methods. The system is flexible with respect to the amount of details the user wants to put into the system: whether all of the steps are to be documented-from the initial sample to the final isotopic or chemical value (e.g. for ${ }^{14} \mathrm{C}$ : drying, acid etching, conversion to $\mathrm{CO}_{2}$, conversion to acetylene, conversion to benzene, LSC counting, etc.) - or if only the final numbers $\left({ }^{14} \mathrm{C}\right.$ sample No. 7 has $35 \mathrm{pMC}$ ) are to be stored, the same data model can be used with just different procedures. 


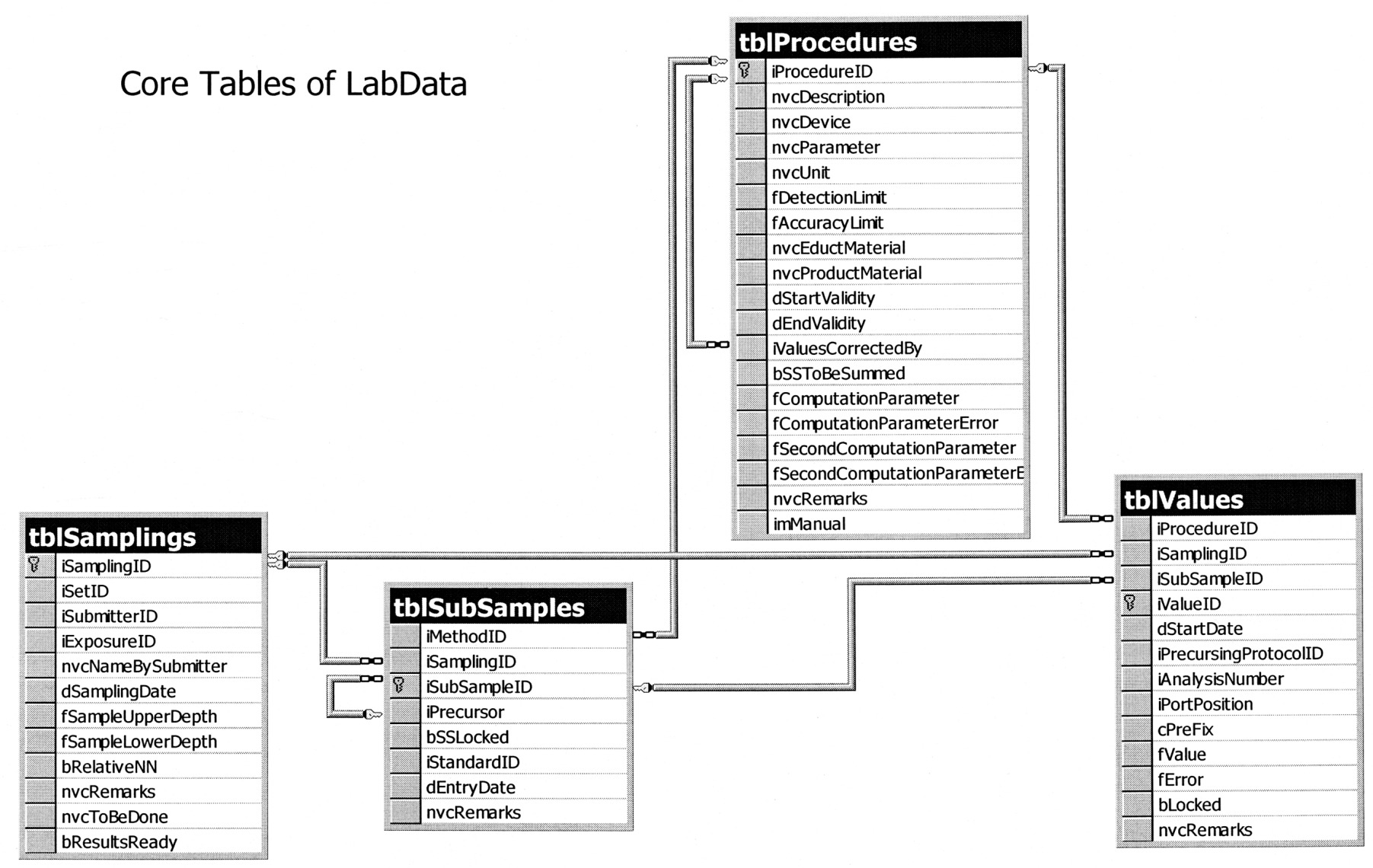

Figure 1 The core of the described data model. The relationships of data records in the subsample table is recursive, allowing generation of subsamples from (sub)samples. 
4. Unique identifiers. Last, but nevertheless important, the data model provides two identifiers that are unique: one (iSamplingID) is unique for the problem as seen from outside the laboratory, which is the point of view of the submitter. The second (iSubSampleID) is unique as seen from inside the laboratory, and identifies the subsample in a specific step of the laboratory process.

One has to be aware that the contents of the tblValues table are not necessarily the final values the submitter wants to receive. In many cases, especially in isotope laboratories, these values are intermediate values. For an enriched tritium sample or a diluted ${ }^{14} \mathrm{C}$ counting gas, the stored values would be the TU of the enriched sample or the pMC of the diluted counting gas. They have to be corrected for at least all the preparation steps between sampling action and last measurement that affect the final result, utilizing the recursion data in the subsample table. All procedures are labeled by a unique iProcedureID in the tblProcedures table (Figure 1). The data model makes no difference between procedures for making measurements (identified by the "iProcedureID" in the tblValues table) and procedures for producing subsamples (identified by the "iMethodID" in the tblSubSamples table). In contrast, the algorithms for calculating mean or corrected values from the raw data have to treat these two kinds of procedures differently: the preparation of a subsample (e.g. a grainsize fraction) by a certain method (e.g. sieving) can generate a unique, related value (e.g. the weight fraction of a specific grain-size range of the total sample). The subsample and this value are identified using the same value for the iMethodID in the tblSubSamples table as for the iProcedureID in the tblValues table. These unique values are of special interest since they yield the numerical relation between the values for a subsample and the value for the precursor (sub)sample. Electrolytic tritium enrichment produces an enriched subsample as well as an isotopic enrichment factor that relates the measured value (in $\mathrm{TU}$ or $\mathrm{Bq} / \mathrm{L}$ ) to the precursor. These values have to be applied as correction factors if the measured values are to be related to the original sample.

Therefore, it is necessary to calculate mean values for the different levels of subsamples. A full example is illustrated in Figure 2: a sediment sample is divided into aliquots, sieved, and analyzed by dissolution and ICP-MS. The arrows to the right in Figure 2 illustrate the flow of data input during laboratory work (production of subsamples and data). Arrows to the left show the steps in the algorithm for extracting the data and relating the data to subsamples. The flag "bSSToBeSummed" in the tblProcedures table (Figure 1) hereby indicates whether the results of different subsamples of one sample have to be added (as in case of the $\mathrm{Pb}$ concentrations of the different grain size fractions) or whether a weighted mean has to be computed (e.g. for the different aliquots).

While this example is a geochemical one, the same principle is applicable to isotope work. In the case of a ${ }^{14} \mathrm{C}$ soil sample, the carbonate fraction, the humic acid fraction and the macrofossil fraction could be subsamples. In this case, the mean value of ${ }^{14} \mathrm{C}$ for the sample also would be computed by the software but probably neglected by the user, since it is of no meaning for the dating problem. The flag "bLocked" in the tblValues table (Figure 1) allows primary values to be excluded from the computation of means that nevertheless should be stored in the database. The flag bSSLocked in the tblSubSamples table is the analog for the subsample recursion: for example, if one of the sieved subsamples in Figure 2 is lost, it would be incorrect to calculate a weighted sum of the remaining subsamples for the $\mathrm{Pb}$ concentration.

Detection limits of a measurement procedure can be defined in the field "fDetectionLimit" in the tblProcedures table. This field describes the final detection limit of a certain measurement device or procedure, as described by Geyh (1972). If this limit is not reached, but the value is nevertheless at the detection limit (e.g. due to shorter counting time), the flag "cPreFix" in the tblValues table is set to "<". The algorithm computing the mean value for a subsample has to keep this flag for the final result, 


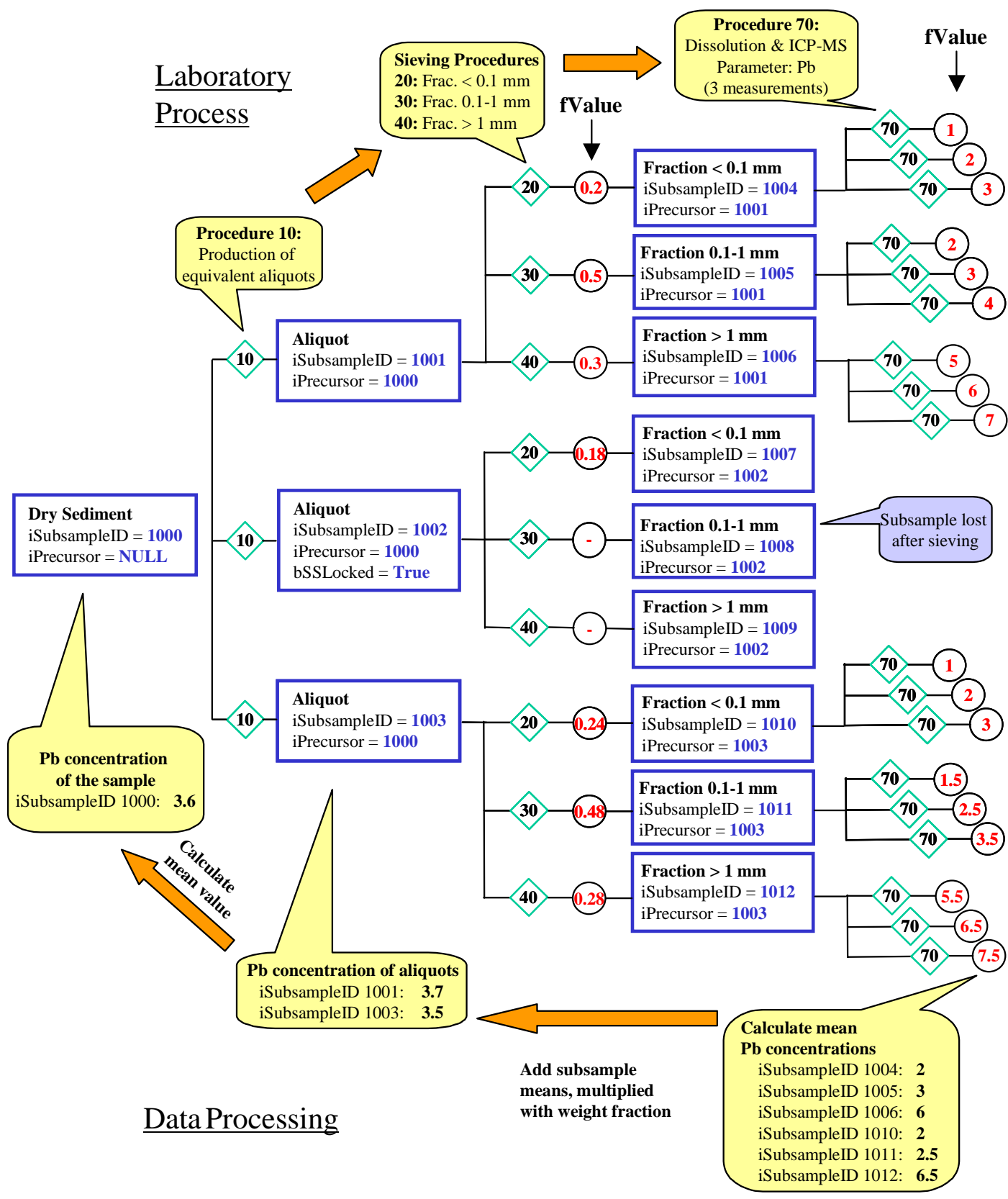

Figure 2 An example of recursion for a soil sample that is divided into aliquots, is sieved to obtain grain-size fractions, which are subsequently analyzed chemically. The arrows to the right indicate data input during laboratory work. Arrows to the left show the steps in the algorithm to relate results for the subsamples to the original sample. 


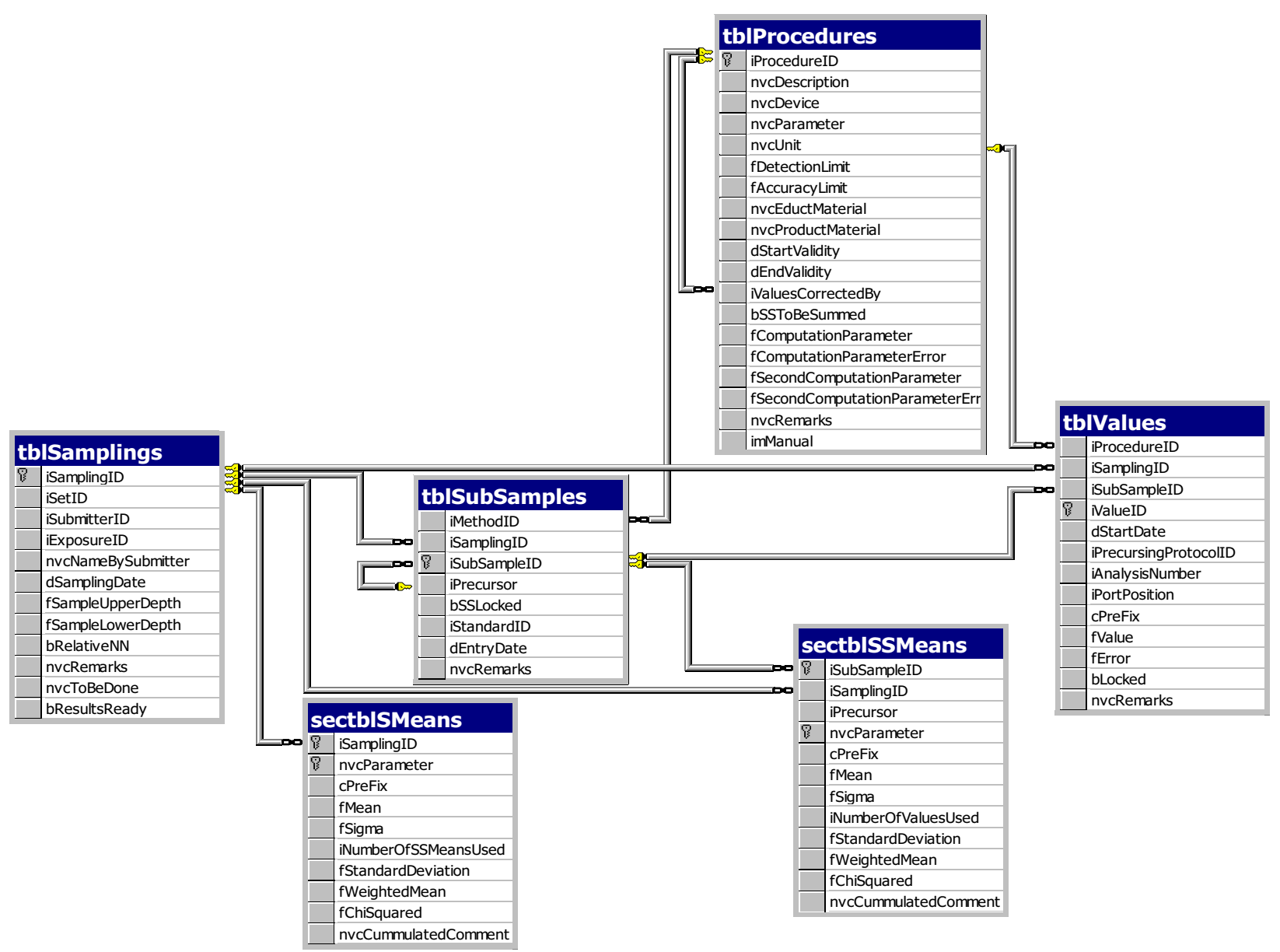

Figure 3 Queries for data have to retrace the subsample recursion. Since this is costly in terms of computing time, mean values are stored in secondary tables. 


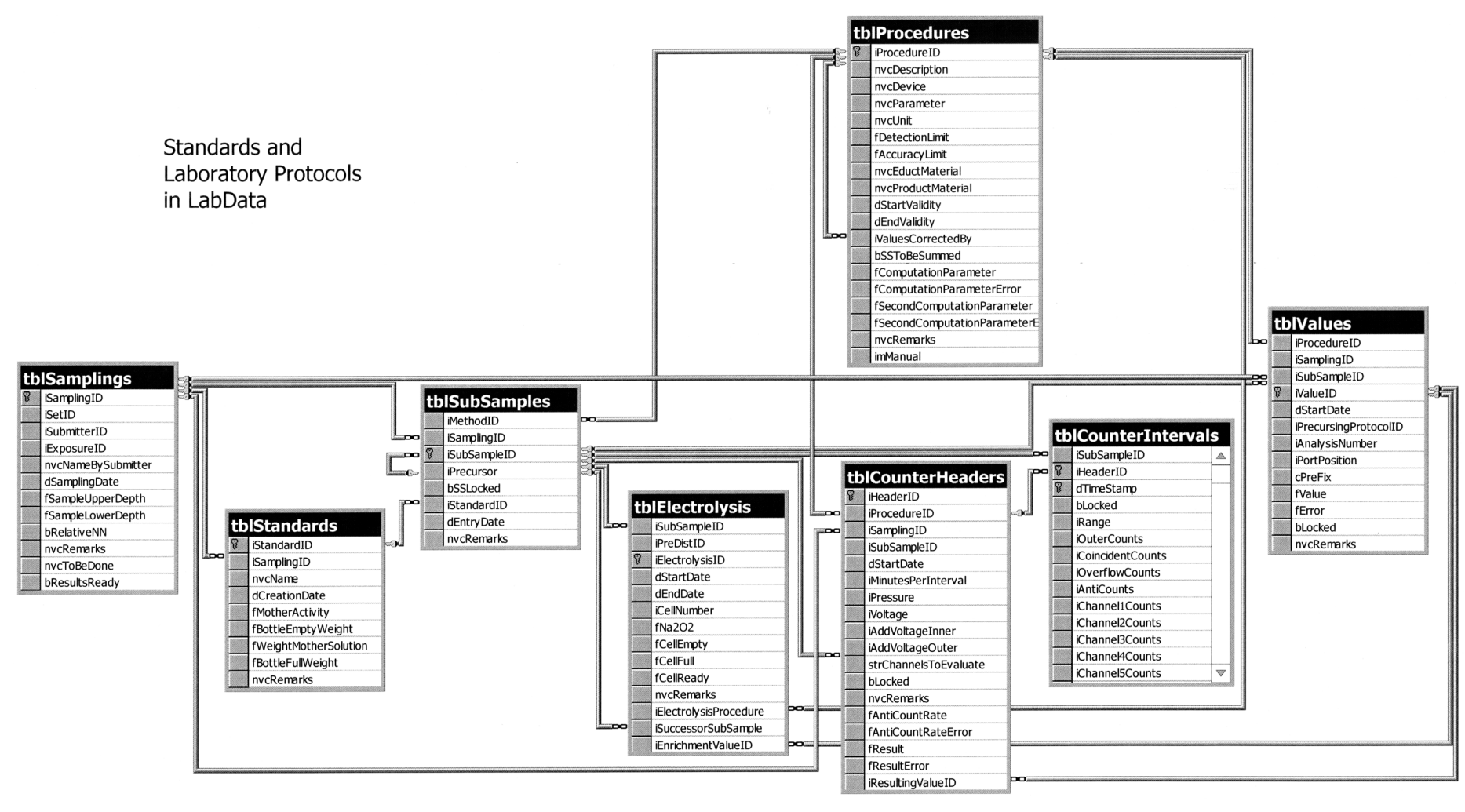

Figure 4 Aliquots of standard material are treated in the same way like subsamples. Laboratory data protocols necessary to calculate intermediate results are stored in own tables. The field "fComputationParameter" in the tblProcedures table allows storage of parameters needed for interactive raw data evaluation (e.g. TU or pMC from raw data of the counters or electrolytic enrichment factors from initial and final weights of water and electrolytic cell). The results of these evaluations are stored in the tblValues table. 
unless a "real" value for the same parameter and subsample exists (e.g. measured with longer counting time or using a different procedure).

\section{Performance}

For most laboratory software packages (Coplen 1998), the data are stored on a database server and the graphical user interface (GUI) runs on a client computer that can be connected to the server by a local area network. If the algorithm for the numerical steps necessary to relate the values to the different subsample levels is implemented in the GUI, computer times for queries are unacceptable. In the first test with numerical algorithms in BASIC (MS Access 97) using two $400 \mathrm{MHz}$ PII computers as server and client, about 50 minutes were needed to obtain an answer to a query for a set of groundwater samples for isotope hydrological analysis from 20 wells involving about 7000 mean values. The reason for this is that the query involved numerous SQL transactions between client and server.

Therefore, we use the database server for the queries and calculations and store the calculated mean values for the parameters for which measurements exist in the database in tables sectblSSMeans and sectblSMeans for the subsamples and samples, respectively (Figure 3). The algorithms to compute the mean values are written as SQL procedures. They are activated using triggers on the table tblValues or during routine database maintenance. Since these procedures exist precompiled on the server within the database file itself, no handshaking is necessary, resulting in faster computations. Using these advantages, the full database maintenance procedure, including setting up a table of roughly 40,000 mean values in table sectblSMeans from >50,000 values in tblValues, together with the relationships between areas, projects and samples (see below) takes less than 15 minutes. Generally only one SQL transaction is then necessary to obtain the desired value(s) for a set of samples, speeding up the initially tested query to less than a minute. A prerequisite of the technique described here is that the data model is implemented using database software able to handle triggers and SQL procedures (e.g., Oracle, RDB, MS SQL server or MS Access 2000, but not MS Access 97 or lower).

\section{Quality Assurance and Laboratory Records}

Laboratory standards, e.g. internal quality assurance standards (blank samples, spikes for electrolytic enrichment, tap water for isotope ratio mass spectrometry) or international standards (ANU, oxalic acid, SMOW, SLAP, GISP), are compiled in a table linked to the sampling table and subsample table (Figure 4). The treatment of the data for quality assurance standards in the data model is identical to that of the data for normal subsamples, since the laboratory processes for both are the same. This directly enables quality assurance control, since the query yielding the reproducibility of a quality assurance standard is identical to that for a normal sample.

In the Hannover Laboratory for Geochronology and Isotope Hydrology, sample preparation is documented using a system of file cards and laboratory books (e.g. date, amounts of reagents used, reaction yields). Since some of these laboratory records contain values needed for the interpretation of the measurement (e.g. dilution factor of the counting gas, the electrolytic tritium enrichment factor), it is useful to store this information in database tables and to carry out the necessary computations within the database (instead of a pocket calculator, for example), even if the algorithm seems very simple.

An example is also given in Figure 4: assume a water sample for tritium analysis got the Ids

SamplingID $=100$ and iSubSampleID $=10000$. With this (sub)sample an electrolytic isotope enrichment (e.g. iProcedureID =5) is performed. The data for this enrichment is entered into an input form in 
the GUI, e.g. the number of the electrolysis cell, the (sub)sample weights necessary for the calculations. In the procedures table (Figure 1) there are fields (e.g. "fComputationParameter") that contain values for the computation: the value of the exponent for the efficiency of cells for electrolytic tritium enrichment is stored here and used during input to compute the enrichment factor. This makes it easy to take into account a change in a measurement parameter value of a device (e.g. change in the value of the exponent for the enrichment efficiency of an electrolysis cell or background and efficiency values for a proportional counter) by just generating a new procedure record. In our example, a new subsample results (iSubSampleID $=20,000$ created newly, iSamplingID $=100$, iPrecursor $=10,000$, iMethodID $=$ 5), and an enrichment value (e.g. of 0.1 corresponding to an isotopic enrichment factor of 10) will be written into tblValues, with iProcedureID $=5$, iSamplingID $=100$, iSubSampleID $=10,000$. Then the enriched subsample (iSubSampleID $=20,000$ ) is converted into a counting gas and measured twice. Again using a GUI form, the raw data of these measurements are imported: a header with data concerning the measurement parameters (e.g. high voltage and gas pressure in the counter) into the tblCounterHeaders table and several cycles with anticoincident counts, guard counts etc. into tblCounterIntervals. Within this GUI form, the measurements are evaluated interactively, resulting in $5 \mathrm{TU}$ and $7 \mathrm{TU}$. Within this evaluation, the fields "fComputationParameter" and "fSecondComputationParameter" in tblProcedures would contain the blank and efficiency values of the proportional counter. Each of the values is stored in tblValues with iSamplingID $=100$ and iSubSampleID $=20,000$. The SQL triggers then compute the weighted mean (in this case $6 \mathrm{TU}$ ) of the tritium value for the enriched subsample (iSubSampleID $=20,000$ ) and the resulting value of $0.6 \mathrm{TU}$ to be attributed to the subsample before enrichment (iSubSampleID $=10,000$ ) and store them in sectblSSMeans. Since the subsample with iSubSampleID $=20,000$ has no precursor and is the only tritium sample from this sampling action, the value of $0.6 \mathrm{TU}$ is also attributed as resulting tritium value to the sampling (iSamplingID $=100$ ) and stored in sectblSMeans.

Many of this is analogous to Coplen's treatment (1998), in which the "penultimate" delta values are imported and related to the SMOW/SLAP scale. It is clear that which laboratory record table is included in the database is dependent on which methods are used in the laboratory.

\section{Old Laboratory Numbers}

Many isotope laboratories have a history of several decades. In the case of the Hannover Laboratory, isotope hydrological and geochronological methods are applied, including proportional counters for tritium and ${ }^{14} \mathrm{C}$, isotope ratio mass spectrometry (IRMS) for ${ }^{18} \mathrm{O}, \mathrm{D}$ and ${ }^{13} \mathrm{C}$, gamma spectrometry for ${ }^{137} \mathrm{Cs},{ }^{228} \mathrm{Ac},{ }^{212} \mathrm{~Pb},{ }^{214} \mathrm{~Pb},{ }^{214} \mathrm{Bi}$ and ${ }^{210} \mathrm{~Pb}$, and thermal ionization mass spectrometry (TIMS) for U/Th dating. More than six different systems of laboratory numbers have been used, in some cases with three identification numbers from three systems for the same groundwater sampling action. To make the invaluable amount of earlier data available without loss of information, the old laboratory identifiers have to be included in any new data model. This is realized in the data model described here using one table and relationship for each old identifier. One has to decide whether the old identification numbers refer to subsamples or the sampling action. The Hv numbers for Hannover ${ }^{14} \mathrm{C}$ measurements identify subsamples, since the humic acid and macrofossil fractions of a single soil sample have different Hv numbers. So the Hv system is linked to the tblSubSamples table. In contrast, the Bh number for tritium referred to the sampling action, and in some cases was used for the results of the stable isotopes ${ }^{18} \mathrm{O}$ and $\mathrm{D}$ as well. So the $\mathrm{Bh}$ system is linked to the tblSamplings table. 


\section{Attribute Data}

When a database system is used as an archive, laboratory identifiers are generally of only minor importance. Typical queries request:

The results for a certain set of samples sent together to a laboratory,

The person or institution submitting samples for inquiries concerning former measurements,

Different samples, evaluated or reevaluated within the context of a project,

Geographic origin of samples: e.g. a certain area or region, a well or sediment core.

Figure 5 shows how the data model was expanded to include the attribute data above the hierarchical level of the tblSamplings table.

The table tblSamplingSets is needed mainly for laboratory administration purposes: samples are sent to a laboratory in sets from one or more sampling campaigns of the submitter, who expects the results by a certain deadline and wants to pay with a single invoice. This table corresponds to the "projects" table of Coplen (1998). The original file of the report sent to the submitter-in Hannover usually MS Word—can be stored in the field "imComment".

A table for the submitter or customer is of course necessary for administrative reasons and is part of nearly every database.

In the case of research laboratories, samples taken for a certain purpose are grouped according to the project involved, but generally taken during different sampling campaigns. As time goes by, earlier results will be reevaluated within the context of a new project: samples taken for an isotope hydrological study in a certain region, for example, could be used for a new project on global paleoclimate or for a study assessing whether a numerical model can be calibrated with isotope data. Hence, a sample can be linked to different projects, or projects can be grouped together in one or more larger projects. A network of m:n relationships between project and sampling data is necessary, in which a project can be part of one or more other projects. This all can be maintained with a single n:m relationship between samples and projects and a recursion within the project table that allows assignment to several projects. The recursion feature of the project table is realized with a string (strSuperiorProjects) in which the IDs of superordinate projects are stored in the form "/\#\#/\#\#\#/\#\#\#\#", where \# are the digits of the superordinate projects (iProjectID). As a simplification for the user, an SQL trigger automatically closes the relationships between samples associated with a project and all superordinate projects.

The geographical information for the samples for which the geographical coordinates are known is stored in the table of exposures ("tblExposures" in Figure 5). This could be a water well or a sediment core or the location of an archeological site. The prerequisite for storing this in a database is a globally unique coordinate system. We preferred a system, which is understandable by humans, and with which computations or at least comparisons like "<" and "> " can be made. Therefore, the UTM and Gauss-Krueger coordinate systems were not suitable: they are easily understandable rectangular systems, but because the reference longitude differs from case to case, they are not necessarily unique, and locations cannot be easily compared numerically. As long as they are related to a unique ellipsoid, one can use degrees in decimal form. We decided to use this approach, stored as floating point numbers and related to the Bessel ellipsoid. This is readable by humans, easily converted to a point on a map, comparisons by ">" and " $<$ " are possible and it is globally unique if the southern and western hemispheres are given negative numbers. Since the original coordinates should be preserved 


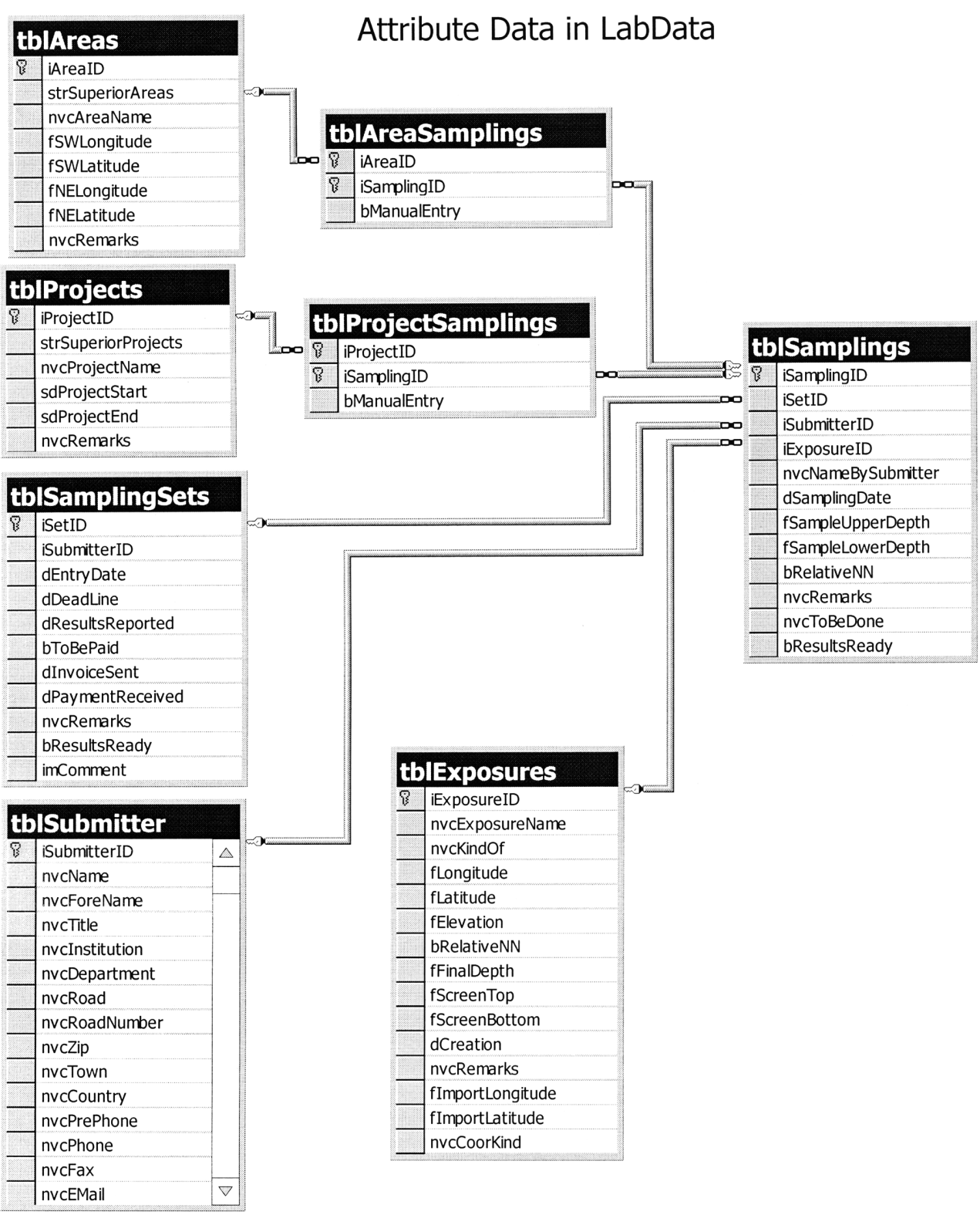

Figure 5 Attribute data describing internal sample sets, submitter, the project context, and geographical origin of the samples.

for the submitter, these are also stored in the fields fImportLongitude and fImportLatitude, but not used for internal computations.

Sample sets are often submitted without exact geographical information about the sampling sites. Nevertheless, "fuzzy" geographical information can exist that might be of future interest (e.g. "a set of samples from the island of Mallorca"). This leads to the definition of areas as a rectangle defined by the coordinates of the southwest and northeast corner in which all the samples in question are sit- 
uated. Especially with a graphical user interface in which the user uses a mouse to delineate a region of interest with "rubberband boxes" on a map, this definition is straightforward. A network of links for areas is obtained, similar to the one for projects, but with relationships that can be numerically defined: there are "rubberband box" areas that completely overlap each other, building a tree-like linkage structure (like Chicago - Illinois - USA), and there are "rubberband box" areas that are situated completely in several superordinate areas that do not overlap completely (for example, Green River - Wyoming - Utah). SQL triggers and procedures were designed that maintain the m:n relationships between samples linked manually to the areas, connecting them to all superordinate areas. This is necessary to find all data in a query on samples from a superordinate area: without coordinates, the set "samples from Mallorca" could otherwise not be found in a search for "samples from Europe". Additional SQL procedures find samples collected at a given exposure and, therefore, have known coordinates, and establish the m:n relationships to all areas the exposure lies in. This saves computing time for a query, since no further coordinate computations are necessary but only a determination of links between tables, for which the database software is optimized. Certainly the definition of areas could be more sophisticated (e.g. with polygonal boundaries following country boundaries), but for practical purposes and fast performance, our method has proved to be more sufficient.

\section{Data Import and Export}

The core data model presented here is in use in the geochemical section of the Federal Institute for Geosciences and Natural Resources (BGR) since nearly a decade. The adaption for geochronology and isotope hydrology in the Institute for Joint Geoscientific Research (GGA), including the secondary mean value computations started in 1997. Meanwhile, the database is used for all techniques in the laboratory for geochronology and isotope hydrology but TIMS U/Th dating. Part of the old data, especially for tritium and ${ }^{14} \mathrm{C}$, was never available in digital form, and only part of it is imported into the system up to now.

More crucial than the import of old data is import and export of data in daily work. Since MS Access allows direct input into the tables on the server, import of digital data can be easily handled using drag \& drop from submitter-supplied spreadsheets and tables, e.g. in Quattro Pro, WordPerfect, MS Excel or MS Word format. In a similar way the GUI allows direct export of raw data and mean values to MS Excel by selecting the needed attribute data, measured parameters and post-processed results. The possibility of direct access especially emphasizes that data integrity has to be kept at the deepest possible level. The SQL constraints, triggers and procedures inhibit the input of data, if the corresponding subsample or sampling does not exist, if a procedure is applied to a subsample of wrong material etc.

\section{Post-Processing Algorithms}

Since the user interface of the database presented here was developed in the Hannover Laboratory for Geochronology and Isotope Hydrology, certain standardized algorithms were included in the code. For sediment cores and depth profiles of radionuclide-excess data, e.g. ${ }^{210} \mathrm{~Pb} /{ }^{226} \mathrm{Ra}$, these algorithms compute the sedimentation rates according to the cf, cic and csr age models (Suckow et al, this issue), or coefficients of bioturbation (Suckow et al. 2001). There are Access-Basic algorithms included, computing groundwater mean residence times using several lumped parameter models (Maloszewski and Zuber 1982, 1996) and model ages corresponding to the $\mathrm{CFCs}, \mathrm{T} /{ }^{3} \mathrm{He}$ or geochemical correction models for ${ }^{14} \mathrm{C}$. The main advantage of having these algorithms in the database system itself instead of in a spreadsheet or another software package, is that there is only one place where bugs in the algorithm module may need to be corrected and no data conversion or even retyping is necessary. Careful and intelligent use is nevertheless a prerequisite: the software will 
always produce a number, but only the user can decide whether the bioturbation or sedimentation rate algorithm is appropriate.

\section{ACKNOWLEDGMENTS}

Beate Burkhart and Klaus Kühne provided some very helpful advice during software development. The English of the manuscript was significantly improved by Clark Newcomb. SQL scripts, including the data definition, triggers and procedures to maintain data integrity, and a graphical user interface based on MS Access, are available free of charge from one of the authors (AS).

\section{REFERENCES}

Coplen TB. 1998. A manual for a laboratory information management system (LIMS) for light stable isotopes. Version 7.0. USGS open file report: 98-284. $124 \mathrm{p}$.

DCSD 1995. Guidelines for data documentation. In: Annual report of the Danish Committee on Scientific Dishonesty 1994. Copenhagen: The Danish Research Councils.

DFG 1998 Empfehlungen der DFG-Kommission "Selbstkontrolle in der Wissenschaft", Vorschläge zur Sicherung guter wissenschaftlicher Praxis. Available at http://www.dfg.de/aktuell/download/ empf_selbstkontr.htm.

Geyh MA. 1972. A comparison: proportional counter and liquid scintillation spectrometer for radiocarbon dating. Proceedings of the VIIIth International Conference on Radiocarbon Dating. Wellington, New Zealand. p 81-93.
Maloszewski P, Zuber A. 1982. Determining the turnover time of groundwater systems with the aid of environmental tracers, I. Models and their applicability. Journal of Hydrology 57:207-31.

Maloszewski P, Zuber A. 1996. Lumped parameter models for interpretation of environmental tracer data. Manual on Mathematical Models in Isotope Hydrogeology, IAEA-TECDOC 10:9-58. Vienna.

Suckow A, Treppke U, Wiedicke M, Weber M. 2001. Bioturbation coefficients of deep-sea sediments from the Peru Basin determined by gamma spectrometry of ${ }^{210} \mathrm{~Pb}_{\mathrm{exc}}$. Deep-Sea Research II 48:3569-92.

Suckow A, Morgenstern U, Kudrass HR. 2001. Absolute dating of recent sediments in the cyclone-influenced shelf area off Bangladesh: comparison of gamma spectrometric $\left({ }^{137} \mathrm{Cs},{ }^{210} \mathrm{~Pb},{ }^{228} \mathrm{Ra}\right)$, radiocarbon and ${ }^{32} \mathrm{Si}$ ages. Radiocarbon. This issue. 